\title{
Group AVTA: A microcomputer system for group voice chronography
}

\author{
JAMES M. DABBS, JR., and THOMAS C. SWIEDLER \\ Georgia State University, Atlanta, Georgia 30303
}

\begin{abstract}
An Apple II microcomputer and electronic interface extends the automatic vocal transaction analyzer (AVTA) approach from dyads to groups, examining the on-off patterns of vocalization among up to eight persons at once. The system eliminates spurious croses talk among microphones, recognizes one person speaking alone, and recognizes several persons speaking at once. Data are included to show how well the system performs with a group of five. On-off patterns of vocalization can be used to investigate differences among groups or among individuals within a group (e.g., in status, dominance, or leadership).
\end{abstract}

On-off patterns of sound and silence in dyadic conversation provide information about the speakers, their relationship to each other, and the nature of their conversation. These patterns have been studied extensively (Feldstein \& Welkowitz, 1978; Jaffe \& Feldstein, 1970), with data usually collected by some version of the automatic vocal transaction analyzer (AVTA; Cassotta, Feldstein, \& Jaffe, 1964). AVTA cancels cross talk between microphones and recognizes when both mem. bers of a dyad are speaking, but it cannot handle the conversation of more than two persons at once. The present paper describes a system that will collect on-off patterns of vocalization from a group of as many as eight persons.

Our approach is modeled after that of Brown (1979), who used computer software for much of the task performed by electronic hardware in AVTA. We are concerned here with data acquisition, with disentangling on-off patterns in the voices of several subjects speaking at once. Equally important problems of managing the data and making statistical comparisons among individuals and groups will not be considered.

\section{ELECTRONIC HARDWARE}

\section{Principle}

Subjects wear lavaliere microphones. The microphones produce signals of a few millivolts, which must be converted to $5 \mathrm{~V}$ before the computer can process them. An electronic hardware device performs all signal conditioning outside the computer. The microphones plug into this device, which filters out noise, amplifies the signals, converts signals above a specified "trigger" level to $5 \mathrm{~V}$, and sends the resulting output to the computer. The circuit diagram for one microphone channel is shown in Figure 1.

Each channel has four amplifiers, A1-A4. Three sets of two capacitors and two resistors, located at the input of $\mathrm{A} 1$, between $\mathrm{A} 1$ and $\mathrm{A} 2$, and at the output of $\mathrm{A} 2$, filter out low- and high-frequency noise and provide capacitive coupling to block the effects of microphone and amplifier dc output bias. The filters have a relatively flat response (less than $2-\mathrm{dB}$ variation) in the 300 to $1,400-\mathrm{Hz}$ range, with $10-\mathrm{dB}$ bandpass cutoff at about $200 \mathrm{~Hz}$ and $3,000 \mathrm{~Hz}$. Amplifiers A1 and A2 increase the approximately 2- to 4-mV microphone output signals to $1.2 \mathrm{~V}$ at the input to $\mathrm{A} 3$, for a gain of about $50 \mathrm{~dB}$. Amplifier gain is adjusted with the potentiometer R1. A3 serves as a comparator; when the input to $A 3$ rises above a trigger level set by the voltage divider $\mathrm{R} 2$, the output from $\mathrm{A} 3$ rises to $5 \mathrm{~V}$. Otherwise, the output is at $-5 \mathrm{~V}$. A diode rectifies the output of $\mathrm{A} 3$ so that only $0 \mathrm{~V}$ or $5 \mathrm{~V}$ goes to the computer. Amplifier A4 drives an LED indicator to show when signals are going to the computer.

\section{Components}

Microphones are of the small "electret" variety. The

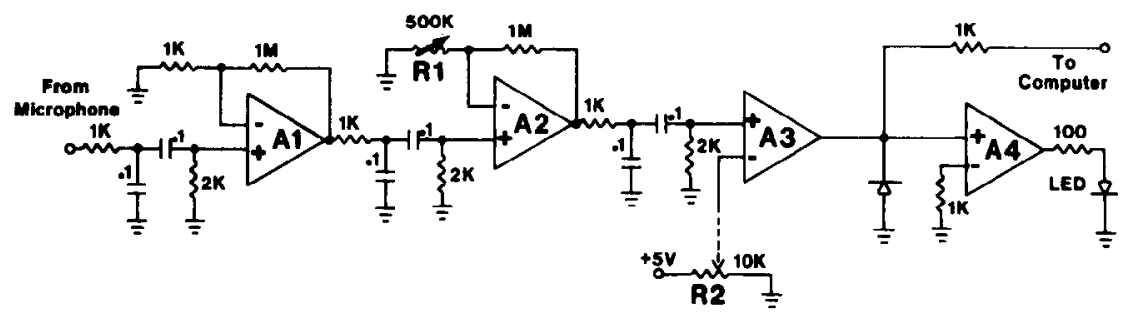

Figure 1. Signal conditioning circuit for one microphone channel. 
microphones are hung around the subject's neck with a cord about 18 in. long. $R 1$ and $R 2$ are miniature circuit board potentiometers; they should be of good quality. The resistors are $1 / 4 \mathrm{~W}$ or $1 / 8 \mathrm{~W}$. Any of a variety of diodes and nonpolarized capacitors may be used. The four amplifiers (A1-A4) for each microphone channel are available in a single integrated circuit (IC), the XR4212. Components can be obtained from Radio Shack, Anchrona Corporation (P.O. Box 2208, Culver City, California 90230), Jameco Electronics (1355 Shoreway Road, Belmont, Califormia 94002), or other sources.

The channels for eight microphones can be assembled on the $14 \times 17 \mathrm{~cm}$ board surface of the PB203A, an adjustable \pm 5 -V power supply from Global Specialties (P.O. Box 1942, New Haven, Connecticut 06509), if resistors and capacitors are used that are physically very small. The high gain of the amplifiers can lead to troublesome interference between the output of one IC and the input of another, unless the ICs are placed in two columns of four each as far apart on the board as possible, with the initial amplifying stages $A 1$ and $A 2$ toward the outside of the board. Microphone jacks can be mounted in holes drilled into the power cord end of the PB203A case, with leads from the jacks run through holes drilled in the face of the PB203A and plugged into the input stages of the eight ICs. Output from the eight ICs goes to the center of the board, where eight lines of a 16-line jumper cable take it on to the computer.

\section{Calibration}

Amplification and trigger level for the eight microphone channels (A-H) must be calibrated before the device will function properly. Calibration is done by first setting the amplification in Channel $A$ and then adjusting the other channels to the same level. Begin by monitoring with an oscilloscope the output of Amplifier A2 in Channel A, immediately before the $1-\mathrm{kohm}$ resistor. While wearing Microphone A, speak loudly and adjust $R 1$ so that the output of $A 2$ is close to $5 \mathrm{~V}$. Signal clipping at $5 \mathrm{~V}$ should be avoided, because clipping indicates that the amplifier cannot discriminate in the upper range of loudness. After Channel $\mathrm{A}$ has been adjusted, use a test signal and a two-channel oscilloscope to set the other channels to the same level. A tape recording of a $500-\mathrm{Hz}$ tone provides a good test signal. Monitor microphone Channels $A$ and $B$ at the inputs to their respective A3s, immediately after the 2-kohm resistors, using a two-channel oscilloscope. Hold Microphones A and $B$ together facing the test tone, with the tone loud enough to produce a signal trace from Channel $A$ of about the same amplitude as the trace produced by a speaking voice. Adjust $R 1$ in Channel $B$ until the oscilloscope traces are the same for Channels A and B. Repeat this procedure with the other channels, adjusting each channel to the level of Channel $A$. Because this adjustment corrects for microphone differences as well as other circuit differences among the eight channels, microphones should be kept with the channels with which they were located when adjustments were made. It is advisable to check adjustment of the amplifiers daily.

Trigger level is adjusted using the voltage divider R2. (Figure 1 shows R2 as part of one channel, but this one component in fact provides the adjustment for all channels; the inverting input of each $A 3$ is tied to the same R2.) Trigger voltage at the input to $A 3$ needs to be high enough that background microphone noise will not rise above it and be scored as voice, and yet low enough to pick up the utterances of a soft-spoken speaker. As with the device of Cassotta et al. (1964), adjustment finally depends upon the judgment of a human operator, who can watch the output of the system and change the trigger level to accommodate unusually loud or soft. spoken groups. In practice, we have always set the trigger level between $.05 \mathrm{~V}$ and $.15 \mathrm{~V}$.

\section{COMPUTER SOFTWARE}

The hardware described above can reveal which microphones are active at a given moment. Further processing is needed to determine which subjects are active, that is, to determine whether a voice heard over a given microphone belongs to the person wearing the microphone or to someone else. This processing is done by an Apple II microcomputer with an SSM parallel interface card (2190 Paragon Drive, San Jose, California 95131) and a Mountain Hardware clock card (300 Harvey West Boulevard, Santa Cruz, California 95060).

\section{Principle}

Intensity of the microphone signals indicates who is speaking and who is silent. Each speaker's voice is louder on his or her own microphone than on the other microphones. When A speaks alone, microphone signals are above the trigger level more often on A's channel than on the other channels. The computer continuously determines which microphone has been above the trig. ger level most often during the preceding $10 \mathrm{msec}$, and it concludes that this microphone belongs to the true speaker. The computer fills in 30-msec pauses within and between a speaker's words (the $300-\mathrm{msec}$ value can be changed in the program).

The situation is more complicated when several subjects speak together. The computer still continuously selects the most active microphone. When it finds very rapid alternation among several microphones, it concludes that the microphones all belong to subjects who are speaking. When people speak together, there is a more or less continuous randomly varying mix of syllables, accents, unvoiced consonants, and pauses, so that even a soft-spoken speaker will have moments of being loudest in a group. The computer picks the most active microphone during each moment, and the same procedure that fills in pauses in the speech of a single 
person will now fill gaps caused by the computer's alternation among the microphones of several simultaneous speakers. Thus while the computer initially selects the most active speaker during each $10-\mathrm{msec}$ interval, it finally produces a continuous record of the speech and silence of all group members.

\section{Program}

A listing of an assembly language program called READPORT is shown in the appendix. This program performs most of the functions of Brown's (1979) electronic audio-to-data converter. It recognizes when several persons speak at once, and it eliminates the spurious sounds from other microphones when one person speaks alone. It stores data in a form convenient for later transfer to a larger computer, in which data can be analyzed using other programs. Data proceed through READPORT in three stages: raw data, interdata, and finished data.

The computer samples raw data from each microphone approximately 8 times $/ \mathrm{msec}$, checking each time to see whether the signal is above the trigger level. At the end of every $10 \mathrm{msec}$, summary raw counts indicate how often each microphone was above the trigger level during the preceding $10-\mathrm{msec}$ interval.

In interdata, summary raw counts are changed to 0 or 1 scores, with 1 assigned to the microphone with the highest count and 0s assigned to the other microphones. This reduces the data for each $10-\mathrm{msec}$ interval from eight separate counts to a single 8-bit byte, with each bit representing the state of one microphone. The most recent 32 bytes (representing $320 \mathrm{msec}$ of realtime) are stored in a temporary interdata buffer. Whenever $1 \mathrm{~s}$ appear in the first and last bytes of this buffer for a given microphone channel, a fill rule changes intervening $0 \mathrm{~s}$ to $1 \mathrm{~s}$, in effect filling in pauses or gaps up to $300 \mathrm{msec}$ long. When a microphone has been quiet for longer than $300 \mathrm{msec}$, a cancel rule changes any 1 surrounded by 0 s to 0 , in effect canceling isolated 10-msec "spikes" of noise. These rules make it easier to hold a voice on than to turn it on: A single 1 can hold the voice on through a brief pause, but more than one 1 is needed to turn it on initially.

Finished data comprise every 25 th byte ( 1 byte every $250 \mathrm{msec}$ ) from the last position of the interdata buffer, after the fill and cancel rules have been applied. Each byte represents the on-off state of all eight microphone channels. Finished data are stored in computer memory and later saved to disk by the operator. A continuous display on the microcomputer screen allows the operator to monitor the system and see how well it is working.

Other investigators may wish to change the program to save the finished data at other intervals or to use different fill and cancel rules. Our 300-msec fill interval and 250-msec sampling interval for finished data are consistent with prior practice in studies of dyadic conversation. Unlike Brown (1979), we have separate rules for turning voices on and holding them on, and we summarize raw counts every $10 \mathrm{msec}$ rather than every $33 \mathrm{msec}$. Our justification for these differences is informal and empirical, based upon a number of hours of monitoring the system with groups of five or fewer subjects. We have explored raw count summary intervals ranging from 5 to $50 \mathrm{msec}$, and $10 \mathrm{msec}$ appears close to optimum. With shorter intervals, the program erroneously attributes sounds to the microphones of subjects who are really silent. With longer intervals (or with more than one " 1 " needed to hold a voice on), the program has difficulty recognizing several speakers at once. The following section presents test data collected using the system as described here.

\section{PERFORMANCE}

Two tests were performed. The first examined the effect of differences in trigger level, and the second examined the ability of the system to handle a group of five speakers at once. Subjects wearing lavaliere microphones and sitting around a 1.2-m-diameter table engaged in simulated conversation, counting aloud or reciting the alphabet at a relatively fast rate of sppech. An experimenter signaled each subject when to start and stop.

\section{Trigger Level}

Variations in trigger level, the voltage level above which amplified microphone signals must rise before being detected as "on," were examined with a group of three subjects. None, one, two, or three of the subjects spoke at once, in all of the eight possible combinations of speakers. The eight combinations were executed in random order, each lasting $25 \mathrm{sec}$. This produced $200 \mathrm{sec}$ of data or, if data are considered separately for each subject, 600 "subject-seconds" of data. The procedure was followed during three sessions, with trigger level set at $.05 \mathrm{~V}, .10 \mathrm{~V}$, and $.15 \mathrm{~V}$ in the three sessions. The data were transferred to a larger computer, which produced a strip-chart representation showing the speech attributed to each subject at each .25 -sec moment.

As trigger level was increased from $.05 \mathrm{~V}$ to $.10 \mathrm{~V}$ to $.15 \mathrm{~V}$, the percent of time speech was attributed to the speaking subjects decreased from $93 \%$ to $86 \%$ to $78 \%$. All subjects paused occasionally to breathe while speaking, and it is our impression that the $93 \%$ figure came close to capturing all the speech. Speech was erroneously attributed to silent subjects . $2 \%$ of the time at the $.05 . \mathrm{V}$ and the $.10 . \mathrm{V}$ levels and none of the time at the $.15-\mathrm{V}$ level. Informal experimentation indicated that as trigger level decreases below $.5 \mathrm{~V}$ and approaches $0 \mathrm{~V}$, the amount of cross talk that is picked up increases enormously. The appropriate trigger level depends upon how much amplification is used, and the level may therefore have to be determined independently by 
different investigators. Because it seems to us more important to eliminate spurious sounds than to pick up all the true sounds, we recommend one begin with a trigger level of $.05 \mathrm{~V}$ or higher.

\section{Five-Person Group}

The system was tested more extensively with a five-person group and the trigger level set at $.10 \mathrm{~V}$. Following the procedure outlined above, data were collected with zero, one, two, three, four, or five persons speaking at once, in all of the 32 possible combinations. The 32 combinations were executed in random order, each lasting $25 \mathrm{sec}$. This produced $800 \mathrm{sec}$ of data or, given the five subjects, 4,000 "subject-seconds" of data (with every subject speaking half the time and silent half the time).

The system seldom erred in attributing speech to subjects who were silent. Of the 2,000 "subject-seconds" in which a subject was silent, sound was detected a total of $15 \mathrm{sec}$, or $.8 \%$ of the time. Amount of speech attributed to subjects who were speaking is shown in Table 1. The system appears able to track any two persons speaking together quite well, but speech is noticeably underestimated when three or more speak together. This tendency appeared to interact with subject differences: Voice attributed to Subjects B and $D$ (presumably the loudest subjects) remained high as number of speakers increased, whereas voice attributed to Subjects A, C, and E dropped off.

The system works well when any member of a group speaks alone or when any one or two members speak at once, but it has difficulty keeping track as more and more people speak. How much this matters will depend upon how often several people actually speak at once and how much detail the investigator needs to know
Table 1

Percent of Time Speech Was Attributed to Each Subject, When Each Subject Spoke With Differing Numbers of Others

\begin{tabular}{lccccc}
\hline & \multicolumn{5}{c}{ Number of Others Speaking } \\
\cline { 2 - 6 } Speaker & 0 & 1 & 2 & 3 & 4 \\
\hline A & 93 & 91 & 79 & 54 & 78 \\
B & 90 & 86 & 93 & 91 & 95 \\
C & 88 & 87 & 67 & 83 & 77 \\
D & 92 & 92 & 92 & 87 & 90 \\
E & 90 & 83 & 77 & 68 & 40 \\
Mean & 91 & 88 & 82 & 71 & 76 \\
\hline
\end{tabular}

about these bursts of "grouptalk." It seems unlikely that several people will speak at once very often or that the investigator will need to follow closely the on-off patterns of individual speakers when several speakers are involved. The system is precise enough for substantive research on group vocalization, and we are using it now to study vocal patterns of leadership in group discussion.

\section{REFERENCES}

Brown, E. F. Apparatus for collecting voice chronography data. Behavior Research Methods \& Instrumentation, 1979, 11, 553557.

Cassotta, L., Feldstein, S., \& Jaffe, J. AVTA: Advice for automatic vocal transaction analysis. Journal of Experimental Analysis of Behavior, 1964, 7, 99-104.

Feldstein, S., \& Welkowitz, J. A chronography of conversation: In defense of an objective approach. In A. W. Siegman \& S. Feldstein (Eds.), Nonverbal behavior and communication. Hillsdale, N.J: Erlbaum, 1978.

Jafre, J., \& Feldstein, S. Rhythms of dialogue. New York: Academic Press, 1970.

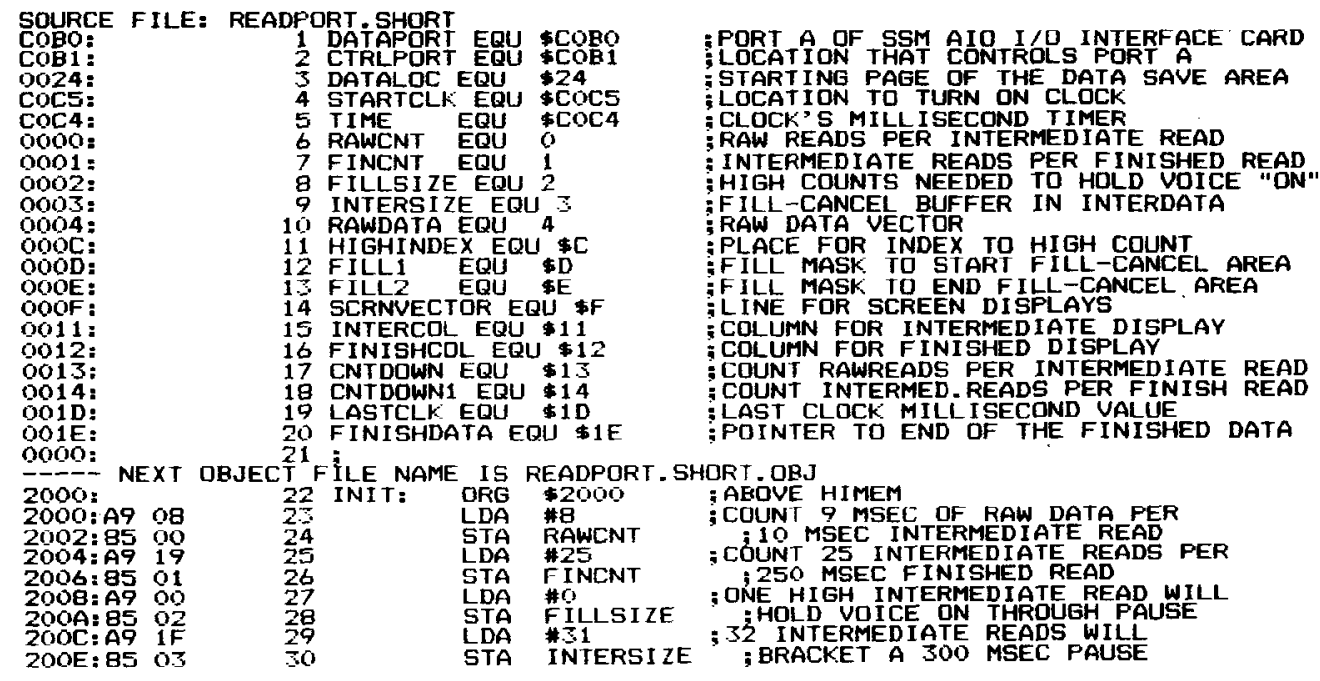




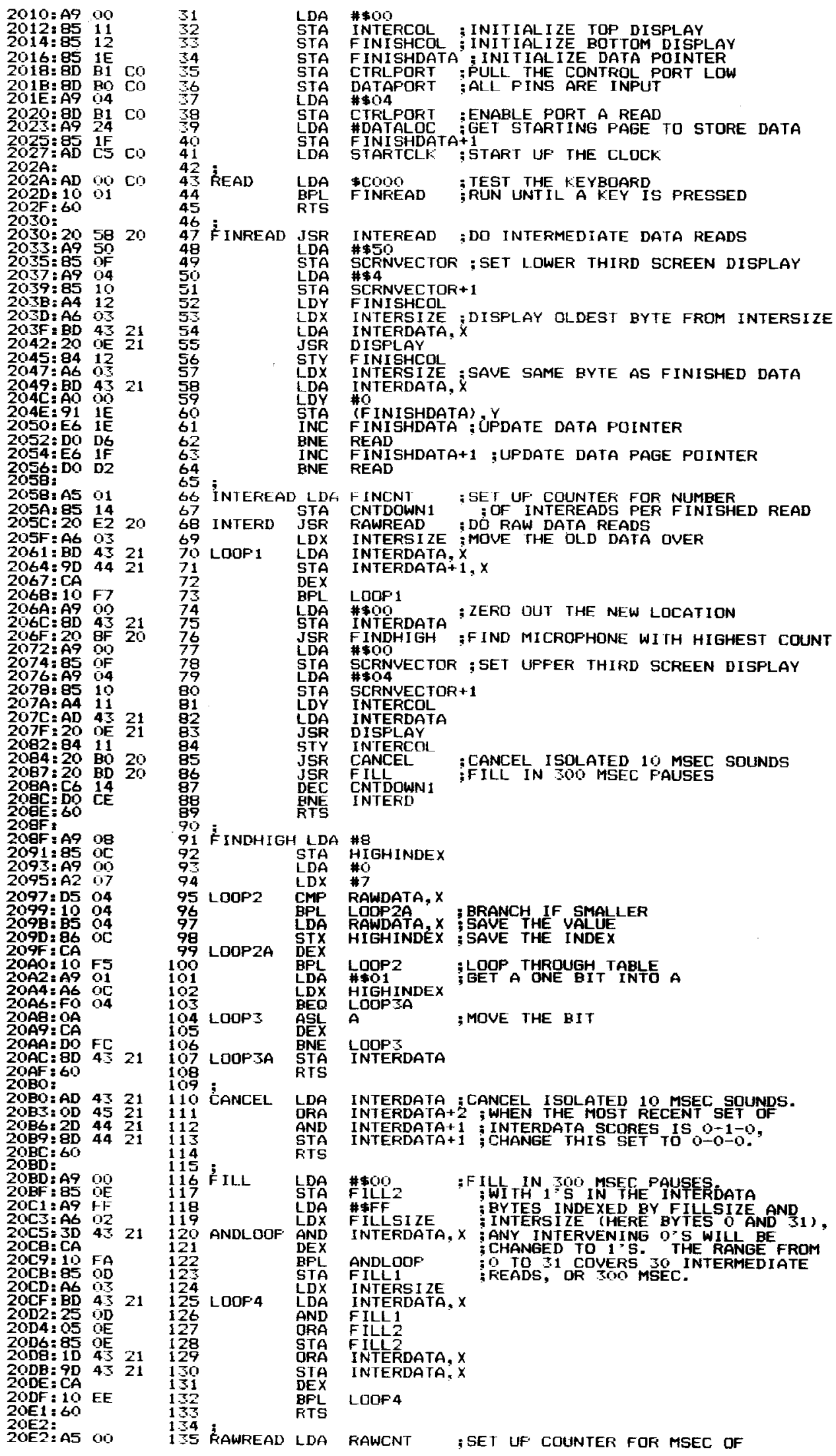




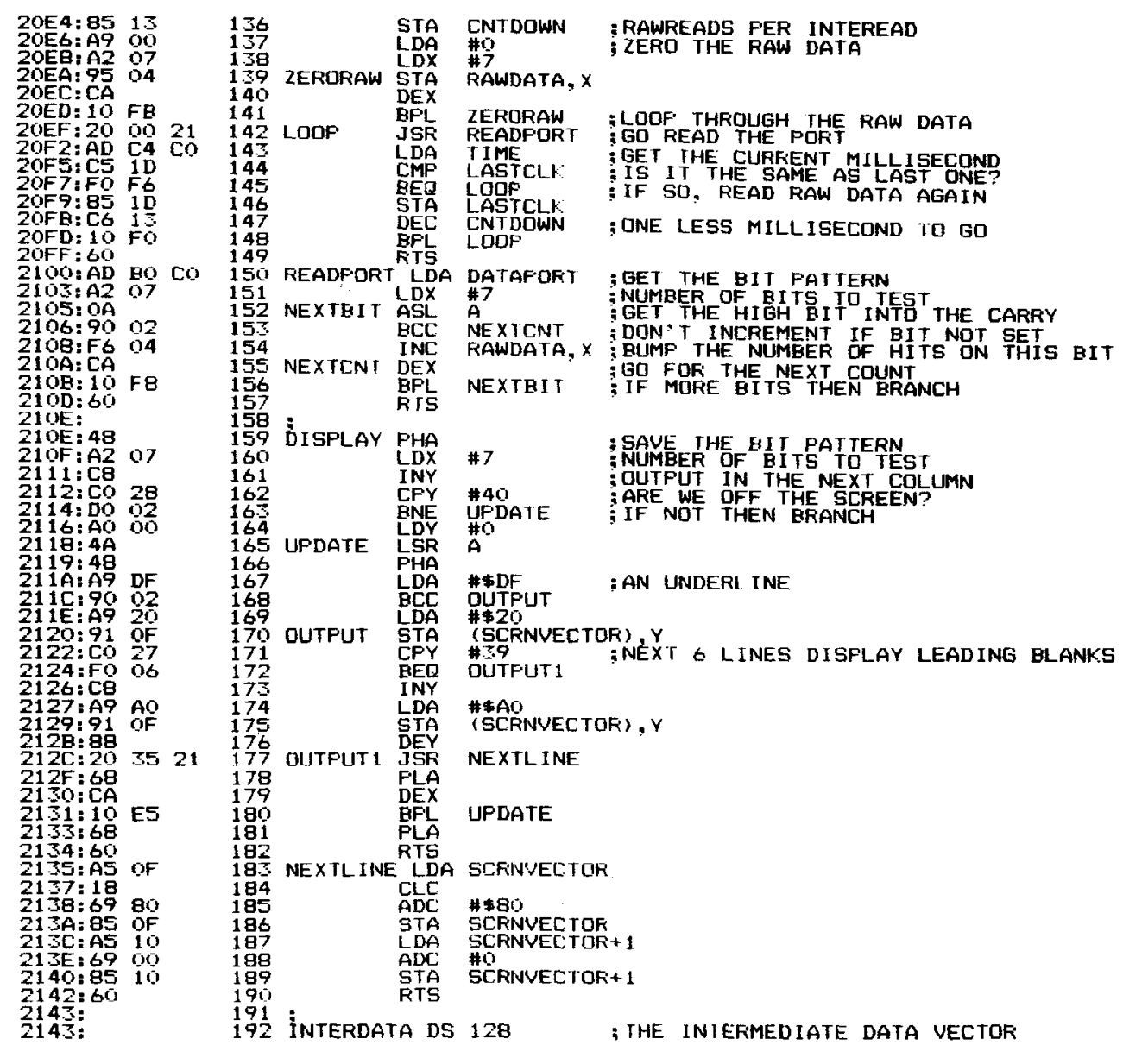

(Received for publication September 29, 1982; revision accepted January 11, 1983.) 